\title{
HIGH-VOLTAGE ELECTRON MICROSCOPY \\ OF THE EARLY STAGES \\ OF RECRYSTALLIZATION IN CuMn
}

\author{
U. KLEMENT, P. HAASEN, P. -J. WILBRANDT \\ Institut für Metallphysik, Universität Göttingen \\ Hospitalstraße 3-7, D-3400 Göttingen, W.-Germany
}

\section{ABSTRACT}

CuMn-specimens were observed by HVEM to obtain information about the recrystallization process influenced by foreign atoms. Up to a concentration of 10 at.\% Mn the SFE of this system does not change. Along the grain boundaries the orientations of the recrystallized grains and of the deformation microstructure were determined to establish the local orientation relationships. The grain orientations can be described by twinning of the deformation microstructure. Only $20-30^{\circ}\langle 111\rangle$ and $40-50^{\circ}\langle 221\rangle$ orientation relationships were found. Known from growth selection experiments these orientation relatioships are already the final ones of the recrystallization texture. Because of the SFE and the large misfit between $\mathrm{Cu}$ and $\mathrm{Mn}$ these orientation relationships were formed rapidly after nucleation. Thus, the orientation formation process is finished after several $\mu \mathrm{m}$ of growth.

\section{INTRODUCTION}

Former recrystallization experiments were motivated by the nucleation problem and the insufficient knowledge of the early stages of the recrystallization process.

The first HVEM-experiments were made on high purity $\mathrm{Cu}$ (99.9998\%) and on Al of lower purity [1]. This allowed to examine the influence of the stacking fault energy (SFE) on the recrystallization process due to its influence on the formation of annealing twins. Unfortunately, the lower purity of the Al-crystals (99.998\%) caused also an 
influence of (precipitated) foreign atoms on the mobility of the grain boundaries. To investigate this influence separately HVEM-experiments in $\mathrm{Cu}$ with different P-contents ( 0.03 at.\% to 0.7 at.\% P) were made [1]. Small additions of $P$ already changed the recrystallization texture without a detectable change of the deformation texture. But at higher P-concentrations the SFE and the deformation texture both changed. In this connection the CuMn system is interessting because additions up to 10 at.\% Mn do not change the SFE [6]. According to Rutter and Aust [5] foreign atoms slow down the motion of general grain boundaries but the mobility of some special boundaries is not affected. Therefore we expect to find them more frequently in CuMn than in $\mathrm{Cu}$. Additionally growth selection experiments (we call them "Beck experiments") are made [2]. They represent a later stage of the recrystallization process. In this way the development of the recystallization process can be observed by determining and comparing the measured OR.

\section{EXPERIMENTAL}

The deformation microstructure (DM) of the CuMn was created by tensile deformation of single crystals grown by Bridgman technique in graphite molds. All specimens were $\langle 110\rangle$-oriented with an accuracy better than $1^{\circ}$. The Mn-concentration was 0.2 at.\% according to chemical analysis. The tensile deformation was made nearly up to fracture $(\sim 60 \%)$ at room temperature. For TEM-investigation slices of the crystals were chemically thinned in a $30 \%$ $\mathrm{HNO}_{3}-70 \%$ methanol solution with small additions of $\mathrm{MnCO}_{3}$.

The structural analysis of the specimens and the in-situ annealing was done in the heating stage of the AEI high votage electron microscope EM 7 of the Max Planck Institut für Metallforschung in Stuttgart. The accelerating voltage was $1000 \mathrm{kV}$, the annealing temperature was about $450-600^{\circ} \mathrm{C}$. The annealing treatment was stopped when the first recrystallized grains were visible in the transparent area. Along the grain boundaries the orientations of the recrystallized grains and of the DM were determined by selected area diffraction (marked by circles on the figures) to establish the local orientation relationships and in this way the character of the grain boundaries. 


\section{THE DEFORMATION MICROSTRUCTURE (DM)}

Compared with earlier experiments on $\mathrm{Cu}$ and $\mathrm{CuP}$ [1] the $D M$ in CuMn is different. It does not show the two components (like matrix and band of secondary slip); i.e. there is no coplanar double slip. In CuMn the final orientation of the tensile axis after deformation was determined to be near $\langle 112\rangle$. Therefore the crystals show single slip as well as some double slip in penetrating slip systems. This seems to be caused by the large misfit of $10 \%$ [7] between $\mathrm{Cu}$ and $\mathrm{Mn}$. Specimens of both kind of DM were prepared. The DM made by single slip can be described by only one orientation. Indeed these specimens needed a higher recrystallization temperature and it is still unknown where and how the recrystallized nuclei originate. The DM made by double slip consists of two orientation types. It should be mentioned that for both DM a considerable spread within the orientation types is visible.

\section{THE RECRYSTALLIZATION MICROSTRUCTURE}

The nucleation of the recrystallized grains happened outside of the transparent area, therefore already grown grains were observed. Generally, one half of the recrystallized grains showed only one orientation over several $\mu \mathrm{m}$ of the transparent area. Some of these grains moved like a tongue into the DM but mostly they were extended parallel to a more or less wavy growth front. The other grains have built-in twin lamellaes or include smaller grains of other orientations. Usually there are two different twin lamellaes built in such a grain. Often, one is nearly perpendicular and one nearly parallel to the growth front.

\section{RESULTS}

For all analysed specimens the OR between the DM and the recrystallized grains is found to be near $20-30^{\circ}\langle 111\rangle$ and $40-50^{\circ}\langle 221\rangle$, respectively. Because of the blurred DM there is also a spread in the calculation of the OR. Nevertheless, the $O R$ can be formed by twinning of the DM. The large misfit between $\mathrm{Mn}$ and $\mathrm{Cu}$ and the unchanged relatively high SFE [8] (compared with CuP [1]) are the reasons for the necessity of 2 or 3 twin operations at most to reach the final (measured) orientation. Those twin operations seem to occur very rapid directly after 
nucleation; in fact they are not visible in the transparent area. In this way the crystal gains quickly an OR of low grain boundary energy. The formation of longer twin chains like in CuP [1] would be energetically disadvantageous.

In Fig. 1 a grain enlarged parallel to the growth front without any twin lamellaes is shown. The DM consists of two orientation types one of which is very blurred. Therefore the OR to this component of the DM varies between $50^{\circ}\langle 764\rangle$ and $60^{\circ}\langle 655\rangle$. The other component of the DM is in $45^{\circ}\langle 763\rangle$ relationship to the recrystallized grain. It can be seen that only one twin operation is needed to create the grain from the first DM-component with corresponding higher or lower misorientation. To obtain the grain orientation from the second orientation type of the DM three twin processes are required. In this context one has to keep in mind that the growth front is very wavy. The grain seems to grow preferable into that orientation type of the DM which leads locally to an OR of smallest grain boundary energy. Obviously there is no need for further twinning.

In Fig. 2 a specimen with including twin lamellaes is shown. The grain is growing into a DM described by only one orientation type with a $40^{\circ}\langle 221\rangle$ relationship. This OR can be achieved from the DM by two twin operations with a misorientation of $8^{\circ}$. To describe the twin lamellaes visible in the recrystallized grain one more twin operation is used. For them a misorientation of $10^{\circ}$ has to be taken into account. Both kinds of twins did not influence the further orientation formation process. Those at the left side are still backtwinned to the grain orientation; the other twin at the right side of Fig. 2 is built in perpendicular to the growth front and therefore is not able to change the grain orientation. Especially this twin lamella is lying in continuation of the dislocation rich layers of the DM. Therefore a dislocation induced process for the twin formation is likely. In this case the Gleiter mechanism is not possible [3].

\section{CONCLUSIONS}

In growth selection experiments on the same material [2] also only two OR $\left(30^{\circ}\langle 764\rangle\right.$ and $\left.48^{\circ}\langle 763\rangle\right)$ were dominating the texture. They are in good correspondence to the OR observed in the HVEM. Specimens of these "Beck experiments" showed also twins but mostly only forward and 
backward twinning between the two final oR were found. Thus, the orientation formation process during recrystallization is already finished after several $\mu_{\mathrm{m}}$ of growth. The final orientations were already achieved in the early stages of recrystallization. This is in contrast to the experiments on $\mathrm{Al}, \mathrm{Cu}$ and $\mathrm{CuP}$ [1]. Indeed, the two exclusively measured $O R$ in CuMn $\left(20-30^{\circ}\langle 111\rangle\right.$ and $40-50^{\circ}\langle 221\rangle$ ) were also found there but obviously, not in that frequency - they are not the dominating OR of these textures. Especially in CuP [1] longer twin chains led to a more complicated texture.

According to Rutter and Aust [5] both $\mathrm{OR}$ can be interpretated as special boundaries. But due to the blurred DM the characterization of the grain boundaries is done here without using the coincidence site model [4]. The misfit and the SFE seem to be the reasons for the differences in the considered systems. To gain a more complete impression of the recrystallization process in presence of foreign atoms one has to solve the question whether the measured $O R$ represent really grain boundaries of low interfacial energy and how the differences between the systems can be described systematically.

\section{ACKNOWLEDEMENT}

Thanks to Prof. Dr. M. Wilkens and the members of the electron microscopy group for the possibility to work at the HVEM of the Max Planck Institut für Metallforschung in stuttgart. The participation at the ICOTOM 9 was enabled by the Doktorandenpreis of the Hoechst AG. The investigations were supported by the Deutsche Forschungsgemeinschaft.

\section{REFERENCES}

1. A. Berger, P.-J. Wilbrandt, F. Ernst, U. Klement and P. Haasen, Prog. Mat. Sci., 32, (1988), 1

2. C. Funke, Diploma Thesis, Göttingen (1990)

3. H. Gleiter, Acta Metall., 17, (1969), 1421

4. M. L. Kronberg and F. H. Wilson, Trans. AIME, 185, (1949), 501

5. J. W. Rutter, K. T. Aust, Acta Metall., 13, (1965), 181

6. Ch. Schwink and Th. Wille, Scripta Met., 14, (1980), 923

7. T. Steffens Ch. Schwink, Acta Metall., 31, (1983), 2013

8. T. Steffens, Ch. Schwink, A. Korner and H. P. Karnthaler, Pil. Mag. A, 56, (1987), 161 


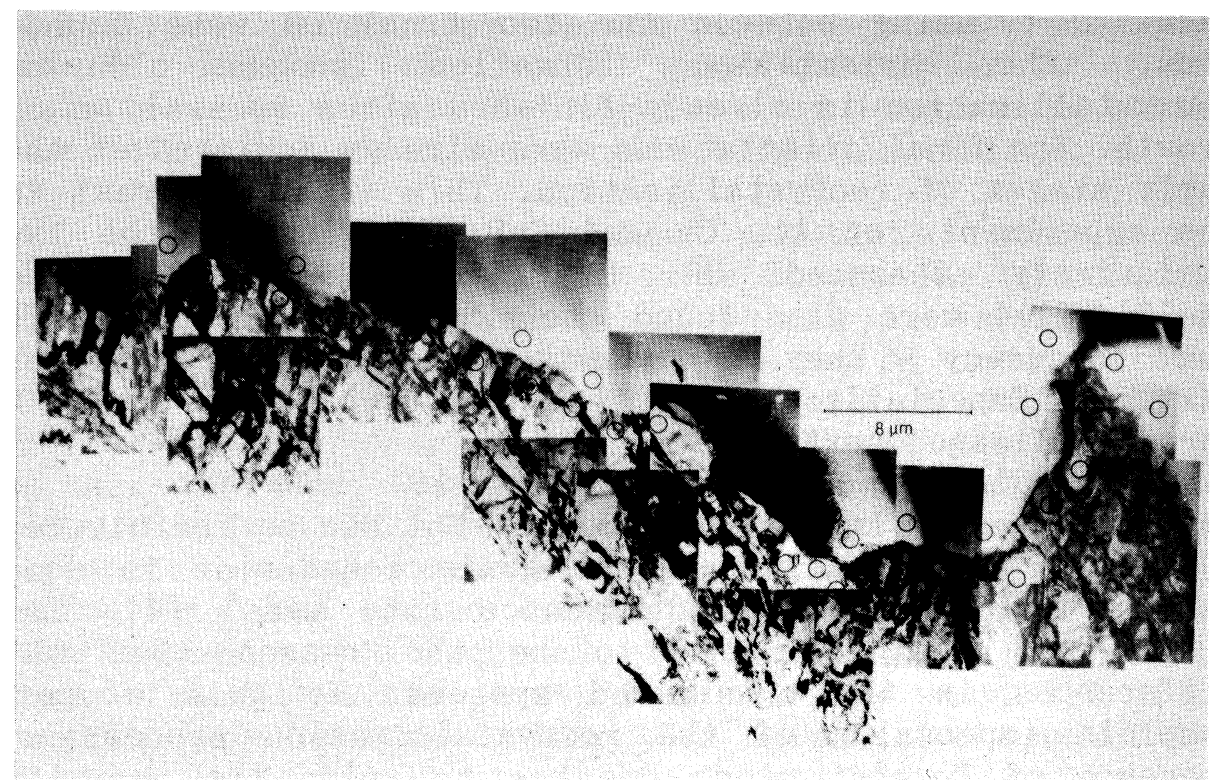

Fig. 1: Microstructure of a recrystallized grain

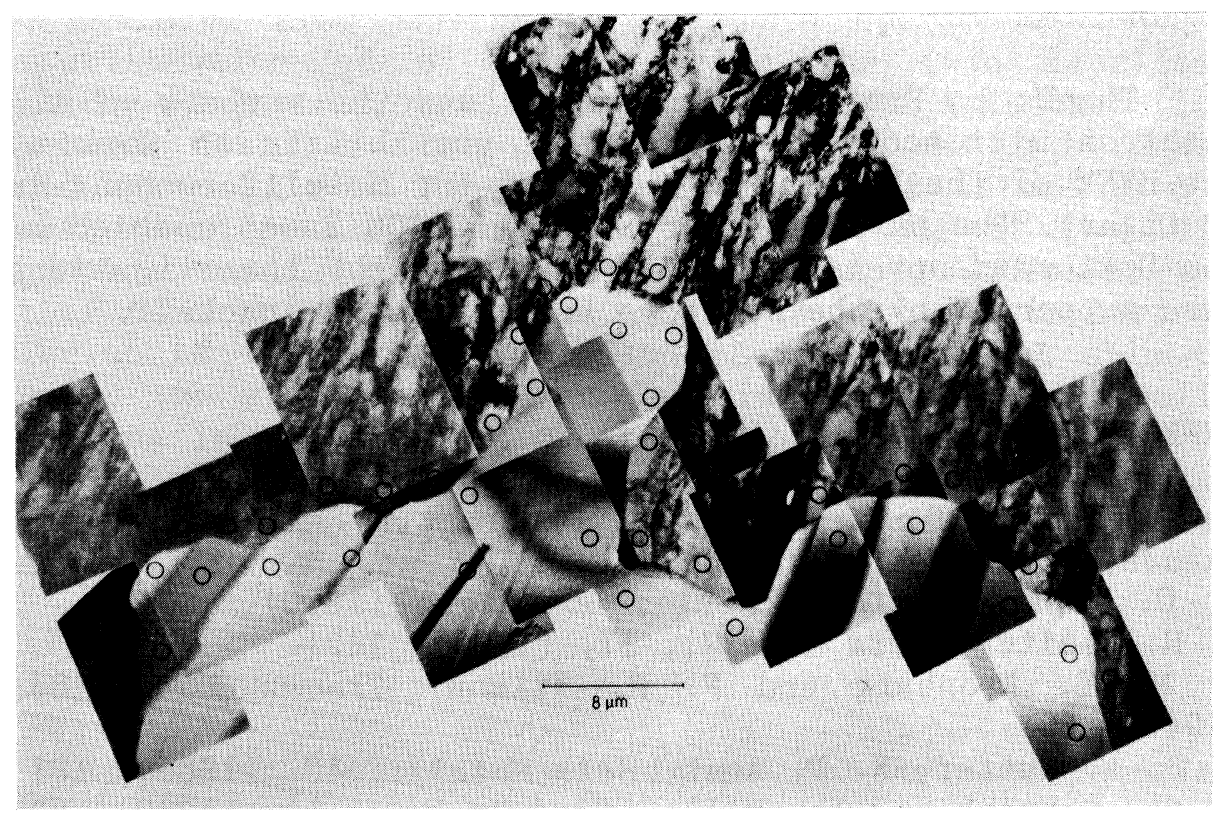

Fig. 2: Microstructure of a recrystallized grain with including twin lamellaes 